\title{
Theory overview of tree-level $B$ decays
}

\section{Fulvia De Fazio*}

Istituto Nazionale di Fisica Nucleare - Sezione di Bari

E-mail: fulvia.defazio@ba.infn.it

I describe the theoretical progress in the study of semileptonic tree-level B decays, and its interplay with recent experimental results. In particular, I focus on two anomalies: the ratios $R\left(D^{(*)}\right)=\frac{\mathscr{B}\left(B \rightarrow D^{(*)} \tau \bar{v}_{\tau}\right)}{\mathscr{B}\left(B \rightarrow D^{(*)} \ell \bar{v}_{\ell}\right)}$ and the inclusive versus exclusive determination of $\left|V_{c b}\right|$. I review a few explanations proposed for such anomalies, and discuss tests to shed light on their origin.

EPS-HEP 2017, European Physical Society conference on High Energy Physics 5-12 July 2017

Venice, Italy

${ }^{*}$ Speaker. 


\section{Introduction}

At present, no new particles have been observed at the LHC, suggesting that maybe the new Physics (NP) scale is not directly accessible, yet. However, a number of anomalies have appeared in indirect searches, analyzing processes where new particles might contribute as virtual states. From this point of view, the most promising channels are the loop-induced ones, suppressed in the Standard Model (SM) and hence more sensitive to NP. However, quite unexpectedly, also treelevel processes manifest some anomalies. Focusing on $B$ and $B_{s}$ decays, observables deviating from SM predictions in loop-induced modes are the angular distributions $P_{5}^{\prime}$ in $B \rightarrow K^{*} \mu^{+} \mu^{-}$[1] and $B_{s} \rightarrow \phi \mu^{+} \mu^{-}$[2], and the ratios $R_{K^{(*)}}=\left.\frac{\mathscr{B}\left(B \rightarrow K^{(*)} \mu^{+} \mu^{-}\right)}{\mathscr{B}\left(B \rightarrow K^{(*)} e^{+} e^{-}\right)}\right|_{q^{2} \in[1,6] \mathrm{GeV}^{2} / \mathrm{c}^{4}}$ [3]. In tree-level decays, deviations have emerged in the ratios $R\left(D^{(*)}\right)=\frac{\mathscr{B}\left(B \rightarrow D^{(*)} \tau \bar{v}_{\tau}\right)}{\mathscr{B}\left(B \rightarrow D^{(*)} \ell \bar{v}_{\ell}\right)}$ [4-6]. The results seem to point to lepton flavour universality (LFU) violation: $R\left(D^{(*)}\right)$ reveals a difference in semitauonic $B$ decays with respect to $\mu$ and $e$ modes, and $R_{K^{(*)}}$ cast a shadow on $\mu-e$ universality. This is in conflict with the SM, where the couplings of the charged leptons to the gauge bosons are the same, and LFU breaking only arises from the Yukawa interaction.

Besides these recent experimental results, there are long-standing puzzles in flavour physics, namely the tension between inclusive and exclusive determinations of $\left|V_{c b}\right|$ and $\left|V_{u b}\right|$ in the CKM matrix. It is tempting to investigate if there is a relation with the above mentioned anomalies. In the following, I shall discuss the deviations related to the semileptonic $b \rightarrow c \ell \bar{v}_{\ell}$ transition. In particular, in Section 2 I shall focus on $R\left(D^{(*)}\right)$, briefly reviewing the experimental situation and a few attempts to address the anomaly. The proposal in [7] will be discussed in some detail, mainly because of the possibility of adopting the same framework to address also the issue of $\left|V_{c b}\right|$ determinations, the subject of Section 3. Conclusions will be presented in the last Section.

\section{Ratios $R\left(D^{(*)}\right)$}

Averaging the measurements of BaBar [4], Belle [5] and LHCb [6] Collaborations, HFAG quotes [8]:

$$
R(D)=0.407 \pm 0.039 \pm 0.024, \quad R\left(D^{*}\right)=0.304 \pm 0.013 \pm 0.07
$$

which deviate from the SM predictions [9]: $R(D)=0.296 \pm 0.016, R\left(D^{*}\right)=0.252 \pm 0.003$ at a global $3.9 \sigma$. Moreover, a very recent result of Belle Collaboration, not included in (2.1), reads $R\left(D^{*}\right)=0.270 \pm 0.035$ (stat) $\pm_{0.025}^{0.028}$ (syst) [10].

The enhancement of the tauonic mode might be explained in two-Higgs doublet models (2HDM) introducing a new doublet of scalars with couplings to fermions proportional to their masses, as for the SM Higgs. However, BaBar showed that it is not possible to simultaneously reproduce $R(D)$ and $R\left(D^{*}\right)$ in the simplest version of 2HDM [4], hence the necessity of a different explanation [11]. Efforts have been devoted to identify models in which the $R_{K^{(*)}}$ anomaly can be also addressed, invoking e.g. the existence of new mediators [13] or of new particles, namely leptoquarks [14]. In most of such models NP mainly couples to the third generation of fermions. Therefore, direct 

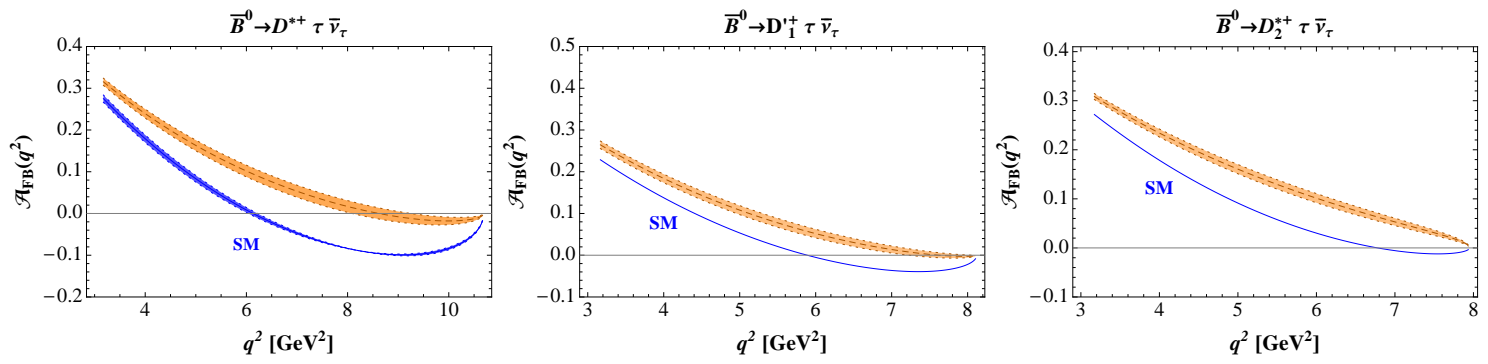

Figure 1: Forward-backward asymmetry $\mathscr{A}_{F B}\left(q^{2}\right)$ for $B \rightarrow D^{*} \tau \bar{v}_{\tau}$ (left panel), $B \rightarrow D_{1}^{\prime} \tau \bar{v}_{\tau}$ (middle) and $B \rightarrow D_{2}^{*} \tau \bar{v}_{\tau}$ (right panel). In each panel the lower curve is the SM prediction, the upper band the NP expectation.

searches of resonances decaying to $\tau^{+} \tau^{-}$at LHC put stringent limits on the masses and couplings of the new mediators [15]. Other constraints emerge from flavour physics, in particular from meson-antimeson mixing [16]. In some NP scenarios such constraints can be safely evaded, for example in models with vector colored leptoquarks [17].

A different, model-independent approach consists in considering all possible structures that can modify the SM effective Hamiltonian describing a given process, without an a priori assumption on the NP identity, and to single out the most sensitive observables to discriminate among the various models. In modes with $\tau$ leptons one can exploit observables sensitive to the lepton mass, unaccessible in the case of muons or electrons [18]. The study in [7] belongs to this research stream. A tensor operator is introduced in the SM effective Hamiltonian for $b \rightarrow c$ semileptonic transitions:

$$
H_{e f f}=\frac{G_{F}}{\sqrt{2}} V_{c b}\left[\bar{c} \gamma_{\mu}\left(1-\gamma_{5}\right) b \bar{\ell} \gamma^{\mu}\left(1-\gamma_{5}\right) v_{\ell}+\varepsilon_{T}^{\ell} \bar{c} \sigma_{\mu v}\left(1-\gamma_{5}\right) b \bar{\ell} \sigma^{\mu v}\left(1-\gamma_{5}\right) v_{\ell}\right]
$$

which affects the ratios $R\left(D^{(*)}\right)$. The new coupling $\varepsilon_{T}^{\ell}$ is assumed to be different for $\ell=\tau$ and $\ell=e, \mu$, namely $\varepsilon_{T}^{e, \mu} \simeq 0$ and $\varepsilon_{T} \equiv \varepsilon_{T}^{\tau}$ [7]. Writing $\varepsilon_{T}=\left|a_{T}\right| e^{i \theta}+\varepsilon_{T 0}$, the data in [4] on $R\left(D^{(*)}\right)$ provide the constraints $\operatorname{Re}\left[\varepsilon_{T 0}\right]=0.17, \operatorname{Im}\left[\varepsilon_{T 0}\right]=0,\left|a_{T}\right| \in[0.24,0.27], \theta \in[2.6,3.7] \mathrm{rad}$.

Predictions for other observables can be obtained varying $\varepsilon_{T}$ in this range, and allow us to discriminate the model from SM and from other NP scenarios. One observable is the forwardbackward asymmetry: $\mathscr{A}_{F B}\left(q^{2}\right)=\left[\int_{0}^{1} d \cos \theta_{\ell} \frac{d \Gamma}{d q^{2} d \cos \theta_{\ell}}-\int_{-1}^{0} d \cos \theta_{\ell} \frac{d \Gamma}{d q^{2} d \cos \theta_{\ell}}\right] / \frac{d \Gamma}{d q^{2}}$, with $q^{2}$ the lepton pair squared invariant mass and $\theta_{\ell}$ the angle between the $\tau$ and $D^{(*)}$ directions in the lepton pair rest frame. The result in Fig. 1 (left plot), which refers to the $D^{*}$ mode, shows that the SM prediction is systematically below the NP result, and has a zero at $q^{2} \simeq 6.15 \mathrm{GeV}^{2}$ shifted towards larger values in the NP model, $q^{2} \in[8.1,9.3] \mathrm{GeV}^{2}$.

The tensor operator also affects semileptonic $B_{(s)}$ decays to excited charmed mesons. The lightest ones of such charmed states, the orbital excitations, are generically indicated as $D_{(s)}^{* *}$ and can be classified in doublets: $\left(D_{(s) 0}^{*}, D_{(s) 1}^{\prime}\right)$ with spin-parity $J^{P}=\left(0^{+}, 1^{+}\right)$, and $\left(D_{(s) 1}, D_{(s) 2}^{*}\right)$ with $J^{P}=\left(1^{+}, 2^{+}\right)$. All these states with and without strangeness have been observed, as discussed in [19]. In the heavy quark limit, the $B \rightarrow D^{* *}$ transitions are described in terms of two universal form factors, $\tau_{1 / 2}(w), \tau_{3 / 2}(w)$. Using their QCD sum rule determination [20] several observables can be computed, in particular the forward-backward asymmetries. Fig. 1 (middle and right panels) 

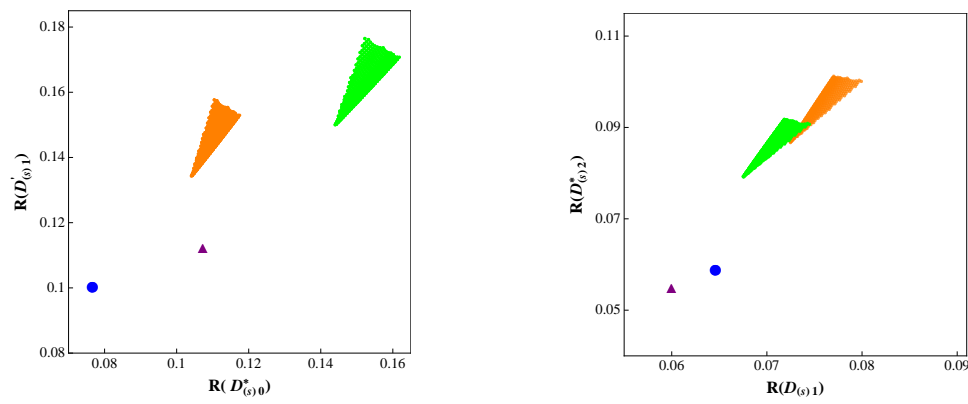

Figure 2: Correlation between $\mathscr{R}\left(D_{(s) 0}^{*}\right), \mathscr{R}\left(D_{(s) 1}^{\prime}\right)$ (left) and $\mathscr{R}\left(D_{(s) 1}\right), \mathscr{R}\left(D_{(s) 2}^{*}\right)$ (right). Orange, dark (green, light) regions refer to mesons without (with) strangeness. The dots (triangles) are the SM results for mesons without (with) strangeness.

shows the asymmetries for $D_{1}^{\prime}$ and $D_{2}^{*}$. The zero of $\mathscr{A}_{F B}\left(q^{2}\right)$, present in both cases in SM, is shifted in $B \rightarrow D_{1}^{\prime} \tau \bar{v}_{\tau}$ to larger values of $q^{2}$, and disappears in $B \rightarrow D_{2}^{*} \tau \bar{v}_{\tau}$. The ratios $\mathscr{R}\left(D_{(s)}^{* *}\right)=$ $\frac{\mathscr{B}\left(B_{(s)} \rightarrow D_{(s)}^{* *} \tau \bar{v}_{\tau}\right)}{\mathscr{B}\left(B_{(s)} \rightarrow D_{(s)}^{* *} \ell \bar{v}_{\ell}\right)}$ can also be computed. The correlations between them are shown in Fig. 2 together with the SM predictions. The effect of the new tensor operator is an enhancement of the ratios, correlated for the two states in the same spin doublet.

\section{Determination of $\left|V_{c b}\right|$ from inclusive and exclusive semileptonic $B$ decays}

The CKM elements $V_{u b}$ and $V_{c b}$ play an important role in the SM description of CP violation in the quark sector. However, the determinations of $\left|V_{u b}\right|$ and $\left|V_{c b}\right|$ from exclusive and inclusive semileptonic $B$ decays are only marginally compatible. For $\left|V_{c b}\right|$ the Particle Data Group quotes [21]

$$
\left|V_{c b}\right|_{\text {excl }}=(39.2 \pm 0.7) \times 10^{-3}, \quad\left|V_{c b}\right|_{\text {incl }}=(42.2 \pm 0.8) \times 10^{-3} .
$$

The tension in (3.1) represents a long-standing puzzle in flavour physics. Within SM a solution has been recently proposed [35], using the Belle Collaboration data for the fully differential $B \rightarrow$ $D^{*} \ell \bar{v}_{\ell}$ decay distribution [36] and scutinizing two different parameterizations of the form factors in the theoretical expression: the Caprini, Lellouch, Neubert (CLN) [31], usually adopted in the experimental analyses, and the one proposed by Boyd, Grinstein and Lebed (BGL) [37]. A similar comparison has been performed for $B \rightarrow D \ell \bar{v}_{\ell}$ [38]. While both form factor parametrizations are based on unitarity and analiticity, the CLN parametrization relies on HQET relations, BGL includes single particle $\left(B_{c}^{*}\right)$ contributions. It is observed that both parametrizations have the same accuracy at large recoil in reproducing the data, but BGL better follows the low-recoil results, with a $\left|V_{c b}\right|$ determination closer to the inclusive one. However, the conclusion about the critical role of the form factor parametrization in the $\left|V_{c b}\right|$ anomaly needs to be validated using different data sets from other experiments. The necessity of improving HQET relations has been stressed, since the uncertainty in the exclusive determination of $\left|V_{c b}\right|$ using the CLN parametrization could be underestimated without the inclusion of the errors on subleading $\Lambda_{Q C D} / m_{Q}$ terms [39].

An intriguing scenario could arise if a common NP solution were found to the $\left|V_{c b}\right|$ puzzle and the $R\left(D^{(*)}\right)$ anomalies. The possibility that NP effects could be responsible of the tension in the 
$\left|V_{c b}\right|$ determinations has been already considered [22-24], with a negative conclusion. In particular, the argument in [23] is that a new scalar or a new tensor operator in the effective $b \rightarrow c$ Hamiltonian, for massless leptons, induces the same effect in both exclusive and inclusive semileptonic modes at zero recoil, producing the same changes in $\left|V_{c b}\right|$. On the other hand, a new vector or axialvector operator would result in modified $W$ couplings and, due to the SM $S U(2)$ symmetry, in $Z$ couplings to fermions modified at a level experimentally excluded [23]. Notice, however, that the inclusive determination of $\left|V_{c b}\right|$ relies on the comparison between the theoretical and experimental full width [25], while exclusive modes are analyzed close to zero recoil [21]. In [26] it has been shown, considering in particular the case of a new tensor operator in the weak Hamiltonian, that the lepton mass is important and, for muons, it can produce a sizable interference between the SM and the NP contribution, with a different impact on the exclusive and the inclusive $B$ decays, hence on $\left|V_{c b}\right|$. This jeopardizes the argument in [23]. The NP effect is different in the inclusive and exclusive modes also in the electron modes, where the SM and NP interference is negligible.

Let us explain the reasoning in [26]. The effective Hamiltonian is in (2.2), with the assumption that not only $\varepsilon_{T}^{\tau}$, but also $\varepsilon_{T}^{(\mu, e)}$ can be non vanishing. The observables are written as the sum of three terms: the SM contribution, the NP term generated by the tensor operator and their interference. For the inclusive mode, the spectrum in the dilepton invariant mass $\hat{q}^{2}=q^{2} / m_{b}^{2}$ reads

$$
\frac{d \Gamma}{d \hat{q}^{2}}=C\left(q^{2}\right)\left[\left.\frac{d \tilde{\Gamma}}{d \hat{q}^{2}}\right|_{S M}+\left.\left|\varepsilon_{T}\right|^{2} \frac{d \tilde{\Gamma}}{d \hat{q}^{2}}\right|_{N P}+\left.\operatorname{Re}\left(\varepsilon_{T}\right) \frac{d \tilde{\Gamma}}{d \hat{q}^{2}}\right|_{I N T}\right]
$$

with $C\left(q^{2}\right)=\frac{G_{F}^{2}\left|V_{c b}\right|^{2} m_{b}^{5}}{96 \pi^{3}} \lambda^{1 / 2}\left(1-\frac{\hat{m}_{\ell}^{2}}{\hat{q}^{2}}\right)^{2}, \hat{m}_{\ell}=m_{\ell} / m_{b}, \lambda=\lambda\left(1, \rho, \hat{q}^{2}\right)$ the triangular function and $\rho=m_{c}^{2} / m_{b}^{2}$. Using the Heavy Quark Expansion (HQE) [27], each term in (3.2) can be written as a series in powers of the inverse heavy quark mass. In turn, each term in the series is the product of coefficient functions times matrix elements of local operators with increasing dimension; at each order in $1 / m_{Q}$, the coefficient functions can be expanded in $\alpha_{s}$. The leading term reproduces the free $Q$ decay width; the $\mathscr{O}\left(m_{Q}^{-1}\right)$ term is absent. The SM result in the case of massive leptons can be found in [28], the NP and INT terms at leading order in $1 / m_{Q}$ is in [7] and at $\mathscr{O}\left(m_{Q}^{-2}\right)$ in [26]. Integrating (3.2) the full width is obtained, so that the branching fraction can be compared to the measurement: $\mathscr{B}\left(B^{+} \rightarrow X_{c} e^{+} v_{e}\right)=(10.8 \pm 0.4) \times 10^{-2}$ [21]. Since no data are separately reported for muon, this result has to be used also in that case, while in the theoretical prediction $m_{e} \neq m_{\mu}$ is kept. The datum constrains $\left(\operatorname{Re}\left(\varepsilon_{T}^{\ell}\right), \operatorname{Im}\left(\varepsilon_{T}^{\ell}\right),\left|V_{c b}\right|\right)$, as shown in Fig. 3, and an upper bound on $\left|V_{c b}\right|$ can be established: at $1 \sigma,\left|V_{c b}\right| \leq 42.85 \times 10^{-3}$ in the muon case and $\left|V_{c b}\right| \leq 42.73 \times 10^{-3}$ in the electron case, in correspondence to the SM point $\varepsilon_{T}^{\ell}=0$. Nonvanishing values of $\varepsilon_{T}^{\ell}$ produce the regions in Fig. 3, which continue to smaller values of $\left|V_{c b}\right|$ if $\left|\varepsilon_{T}^{\ell}\right|$ is increased. $\left|V_{c b}\right|$ is bounded from below by the $B \rightarrow D^{(*)} \ell \bar{v}_{\ell}$ modes. The description of such modes requires the $B \rightarrow D^{(*)}$ form factors that, in the HQ limit, can be related to the Isgur-Wise function $\xi(w)$ [29], with $w$ defined by the relation $q^{2}=m_{B}^{2}+m_{D^{(*)}}^{2}-2 m_{B} m_{D^{(*)}} w .1 / m_{Q}$ and $\mathscr{O}\left(\alpha_{s}\right)$ corrections can be included [30,31]. For finite quark mass, several form factor determinations are available. In [26] lattice QCD results $[32,33]$ have been exploited. The other form factors have been derived using HQ relations at NLO, as described in [7]. For the decays $B^{-} \rightarrow D^{0} \ell^{-} \bar{v}_{\ell}$, the BaBar Collaboration has provided separate measurements for $\mu$ and $e$ modes [34]: $\mathscr{B}\left(B^{-} \rightarrow D^{0} \mu^{-} \bar{v}_{\mu}\right)=(2.25 \pm 0.04 \pm 0.17) \%$, $\mathscr{B}\left(B^{-} \rightarrow D^{0} e^{-} \bar{v}_{e}\right)=(2.38 \pm 0.04 \pm 0.15) \%$. Comparison of these data with theory constrains 

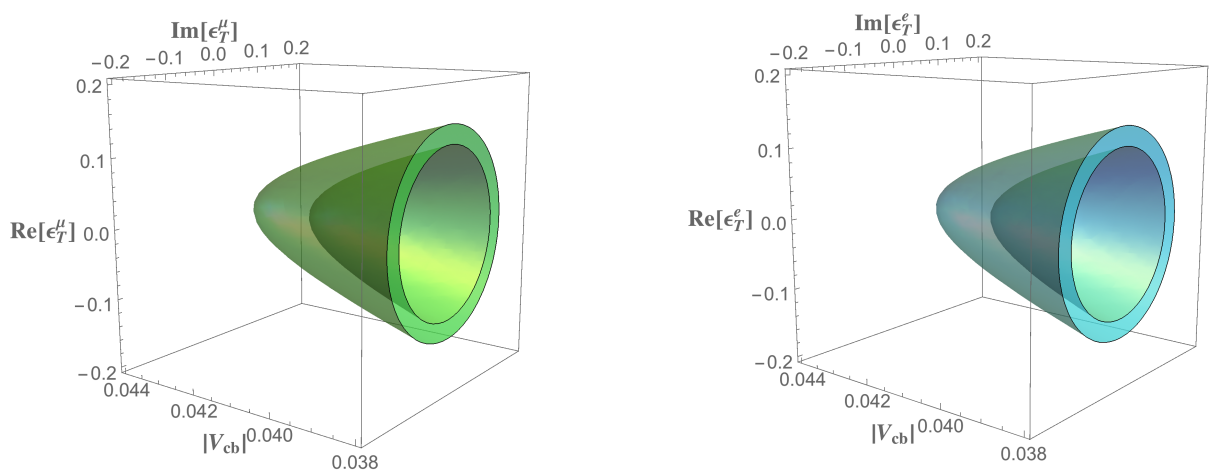

Figure 3: Cutaway view of the parameter space $\operatorname{Re}\left(\varepsilon_{T}^{\ell}\right), \operatorname{Im}\left(\varepsilon_{T}^{\ell}\right)$, and $\left|V_{c b}\right|$ allowing to obtain $\mathscr{B}\left(B^{+} \rightarrow\right.$ $X_{c} \ell^{+} v_{\ell}$ ) within $1 \sigma$ for muon (left) and electron mode (right).

$\left(\operatorname{Re}\left(\varepsilon_{T}^{\ell}\right), \operatorname{Im}\left(\varepsilon_{T}^{\ell}\right),\left|V_{c b}\right|\right)$, while for $B^{-} \rightarrow D^{* 0} \ell^{-} \bar{v}_{\ell}$, the comparison is performed for the distribution $\frac{d \Gamma}{d w}$ close to the zero recoil point $w \rightarrow 1$ using the BaBar result [34].

All the constraints from inclusive and exclusive modes can be combined. In the muon channels, the upper (left and middle) panels of Fig. 4 show the parameter regions selected by the exclusive constraints (larger yellow space) superimposed to the region determined from the inclusive mode (smaller inner space). The left plot refers to $\mathscr{B}\left(B^{-} \rightarrow D^{0} \mu^{-} \bar{v}_{\mu}\right)$, the middle one to $\frac{d \Gamma}{d w}\left(B^{-} \rightarrow D^{* 0} \mu^{-} \bar{v}_{\mu}\right)$. The region where all constraints are fulfilled is shown in the right plot. The bottom panels in the same figure refer to the electron mode.

In the case of $\mu$, exclusive data put a lower bound on $\left|V_{c b}\right|$ and slightly reduce the upper
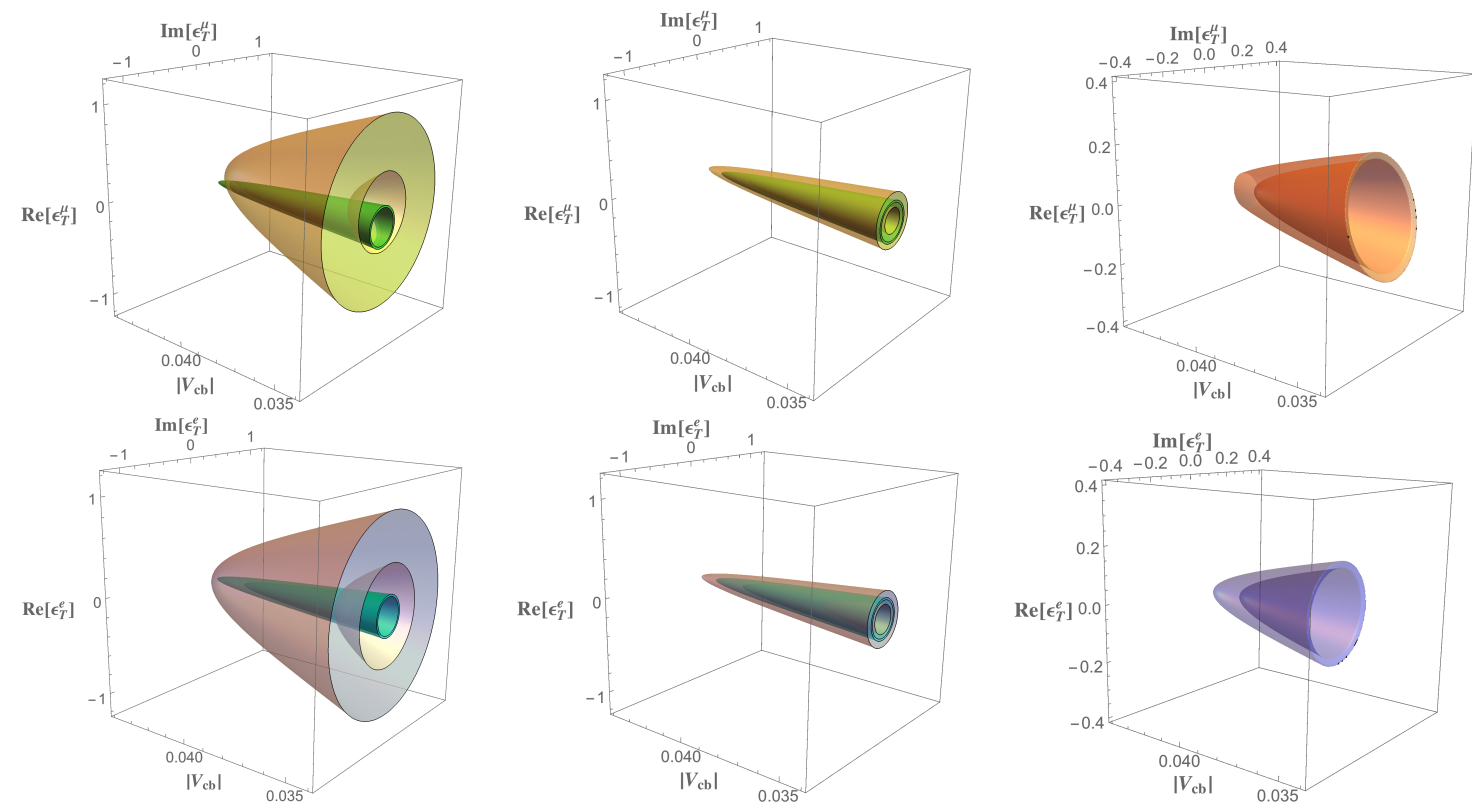

Figure 4: Top panels: allowed regions in the $\left(\operatorname{Re}\left(\varepsilon_{T}^{\mu}\right), \operatorname{Im}\left(\varepsilon_{T}^{\mu}\right),\left|V_{c b}\right|\right)$ parameter space, determined from the inclusive mode (internal hollow region) together with the exclusive $D$ mode (external hollow region, right plot), and from the inclusive mode together with the decay to $D^{*}$ (external hollow region, middle plot). The intersection of the three regions is shown in the right plot. The bottom panels refer to the electron case. 
bound, as shown in Fig. 5 (left and middle plots). The figure displays the projections in the $\left(\operatorname{Re}\left(\varepsilon_{T}^{\mu}\right), \operatorname{Im}\left(\varepsilon_{T}^{\mu}\right)\right)$ plane of the parameter space in correspondence to the extreme values of $\left|V_{c b}\right|$, those for which the regions selected using the various constraints do not overlap. The obtained range is $\left|V_{c b}\right| \in[0.0343,0.0421]$. The range for $\varepsilon_{T}^{\mu}$ depends on $\left|V_{c b}\right|$, with the largest allowed value $\left|\varepsilon_{T}^{\mu}\right| \simeq 0.2$. The lepton mass effect and the interference term are at the origin of the fact that the symmetry axes of the two regions of parameters, resulting from the inclusive and the exclusive constraints, do not intersect the $\left(\operatorname{Re}\left(\varepsilon_{T}^{\mu}\right), \operatorname{Im}\left(\varepsilon_{T}^{\mu}\right)\right)$ plane at the origin and do not coincide. Hence, the NP contribution differently affects the inclusive and the two exclusive channels.

For the electron mode, changes in the results are due to the tiny value of the $e$ mass. The exclusive constraints do not modify the upper bound $\left|V_{c b}\right| \leq 0.04273$ put by the inclusive analysis, and a lower bound is found, as shown in Fig. 5 (right). The range $\left|V_{c b}\right| \in[0.036,0.0427]$ and the bound $\left|\varepsilon_{T}^{e}\right| \leq 0.17$ are found. The conclusion is that it is possible to find sets of three parameters fulfilling all the constraints both for $\mu$ and $e$. For $\left|V_{c b}\right|$ this happens in the range $\left|V_{c b}\right| \in[0.036,0.042]$. Although the uncertainty on $\left|V_{c b}\right|$ is quite sizable, the analysis shows that it is possible to reconcile its inclusive and exclusive determinations via a non SM contribution.

The role of the NP contribution and of the interference between SM and NP can be assessed integrating separately the three terms in (3.2). The results, divided by the full decay width, are denoted by $\mathscr{B}_{S M}, \mathscr{B}_{N P}, \mathscr{B}_{I N T}$. Fig. 6 shows the ratios $\frac{\mathscr{B}_{I N T}}{\mathscr{B}_{N P}}$ and $\frac{\mathscr{B}_{I N T}+\mathscr{B}_{N P}}{\mathscr{B}_{S M}}$ obtained varying $\left(\operatorname{Re}\left(\varepsilon_{T}^{\ell}\right), \operatorname{Im}\left(\varepsilon_{T}^{\ell}\right),\left|V_{c b}\right|\right)$ in the allowed ranges. The left plots refer to the inclusive modes, the right ones to the exclusive decay to $D$. Green color refers to the muon, blue to the electron. $\mathscr{B}_{I N T}$ can be sizable with respect to $\mathscr{B}_{N P}$ for the muon and is negligible for the electron. For both $\mu$ and $e, \mathscr{B}_{I N T}+\mathscr{B}_{N P}$ is negligible for large $\left|V_{c b}\right|$ and sizable for small $\left|V_{c b}\right|$. The tensor operator has a larger impact in the inclusive mode than in $D$ channel. NP affects in a different way these decays: this is an example of how a new term in the effective Hamiltonian could be at the origin of the $\left|V_{c b}\right|$ anomaly.
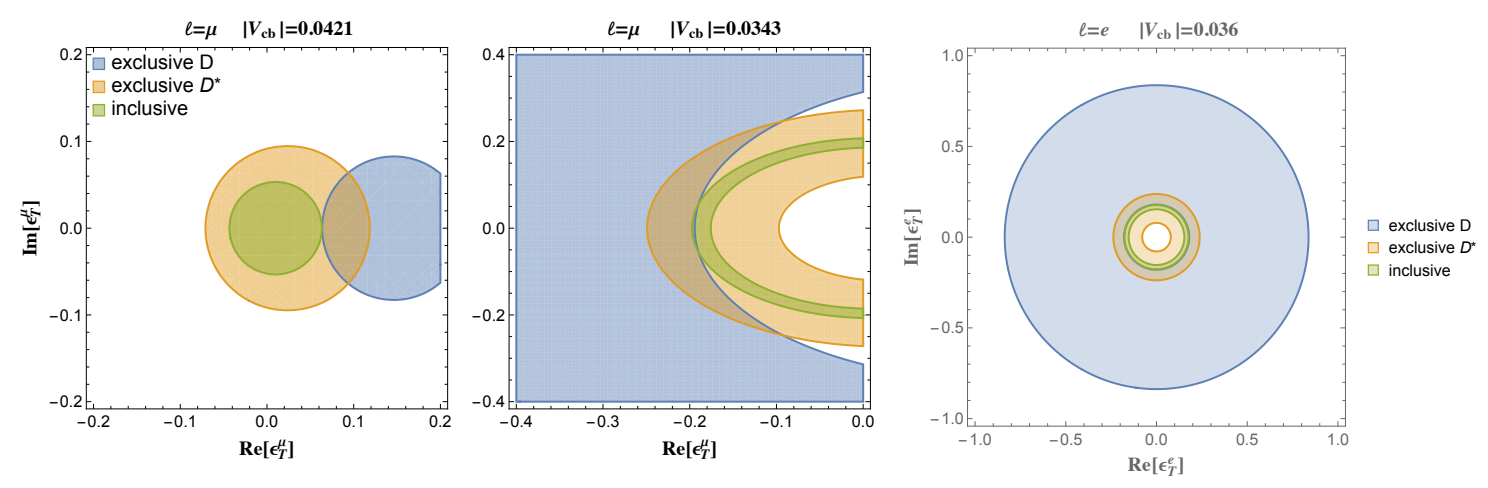

Figure 5: Muon channel (left and middle panel): projections of the overlap region in Fig. 4 on the $\left(\operatorname{Re}\left(\varepsilon_{T}^{\mu}\right), \operatorname{Im}\left(\varepsilon_{T}^{\mu}\right)\right)$ plane. The blue region corresponds to the constraint from the exclusive decay to $D$, the orange region to the constraint from $D^{*}$ and the green region to the constraint from the inclusive mode. The left (middle) plot corresponds to the largest (smallest) allowed value of $\left|V_{c b}\right|$. Electron channel (right plot): projections of the overlap region in Fig. 4 in the $\left(\operatorname{Re}\left(\varepsilon_{T}^{e}\right), \operatorname{Im}\left(\varepsilon_{T}^{e}\right)\right)$ plane. $\left|V_{c b}\right|$ is set to the smallest allowed value. Color coding as in the $\mu$ case. 

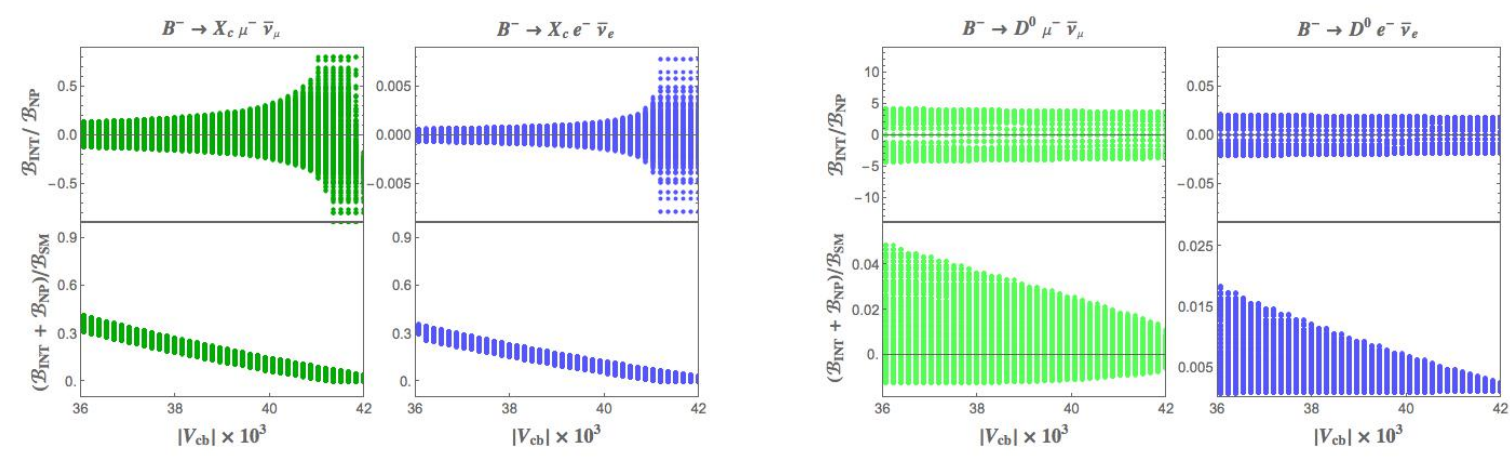

Figure 6: Relative size of the NP contributions to $\mathscr{B}\left(B^{-} \rightarrow X_{c} \mu^{-} \bar{v}_{\mu}\right)$ (left panel, green) and $\mathscr{B}\left(B^{-} \rightarrow\right.$ $\left.X_{c} e^{-} \bar{v}_{e}\right)$ (blue), and to $\mathscr{B}\left(B^{-} \rightarrow D^{0} \mu^{-} \bar{v}_{\mu}\right)$ (right panel, green) and $\mathscr{B}\left(B^{-} \rightarrow D^{0} e^{-} \bar{v}_{e}\right)$ (blue).

\section{Conclusions}

The anomalies emerged in $B$ decays seem to point to LFU violation. Several paths can be followed to confirm/discard this conclusion. The $R\left(D^{(*)}\right)$ results suggest to search similar effects in $B_{s}, B_{c}, \Lambda_{b}$ semileptonic decays, as well as in $B$ to $D^{* *}$ modes. The purely leptonic $B_{c} \rightarrow \tau \bar{v}_{\tau}$ channel is a testing ground for the scenario with a new tensor operator. The analyses would also contribute to shed light on the $\left|V_{c b}\right|$ puzzle: in this case, separate rmeasurements for inclusive and exclusive semileptonic $B$ to $\mu$ and $e$ modes are called for [26]. To check the impact of form factor models, it is important to investigate if there are analogous hints of LFU violation in $b \rightarrow u$ transitions, also in connection with the determination of $\left|V_{u b}\right|$.

Acknowledgments. I thank J. Albrecht, D. Becirevic and P. Urquijo for inviting me to present this talk at the EPS-HEP 2017 conference, and P. Biancofiore and P. Colangelo for collaboration on the topics discussed here. The work has been carried out within the INFN project QFT-HEP.

\section{References}

[1] R. Aaij et al. [LHCb Collaboration], JHEP 1602 (2016) 104.

[2] R. Aaij et al. [LHCb Collaboration], JHEP 1307 (2013) 084.

[3] R. Aaij et al. [LHCb Collaboration], Phys. Rev. Lett. 113 (2014) 151601.

[4] J. P. Lees et al. [BaBar Collaboration], Phys. Rev. Lett. 109 (2012) 101802; Phys. Rev. D 88 (2013) 072012.

[5] M. Huschle et al. [Belle Collaboration], Phys. Rev. D 92 (2015) 072014; A. Abdesselam et al. [Belle Collaboration], arXiv:1603.06711 [hep-ex].

[6] R. Aaij et al. [LHCb Collaboration], Phys. Rev. Lett. 115 (2015) 111803 Erratum: [Phys. Rev. Lett. 115 (2015) 159901]; arXiv:1708.08856 [hep-ex], and C. Bozzi, these proceedings.

[7] P. Biancofiore, P. Colangelo and F. De Fazio, Phys. Rev. D 87 (2013) 074010.

[8] Y. Amhis et al., arXiv:1612.07233 and online update at http://www.slac.stanford.edu/xorg/hflav

[9] S. Fajfer, J. F. Kamenik and I. Nisandzic, Phys. Rev. D 85 (2012) 094025. 
[10] S. Hirose et al. [Belle Collaboration], arXiv:1709.00129 [hep-ex].

[11] A. Datta et al., Phys. Rev. D 86 (2012) 034027.

[12] A. Crivellin et al., Phys. Rev. D 86 (2012) 054014; D. Becirevic et al., Phys. Lett. B 716 (2012) 208; M. Tanaka et al., Phys. Rev. D 87 (2013) 034028; A. Celis et al., JHEP 1301 (2013) 054; Y. Sakaki et al., Phys. Rev. D 88 (2013) 094012; D. Bardhan et al., JHEP 1701 (2017) 125; S. Iguro and K. Tobe, arXiv:1708.06176 [hep-ph].

[13] A. Greljo, G. Isidori and D. Marzocca, JHEP 1507 (2015) 142.

[14] B. Bhattacharya et al., Phys. Lett. B 742 (2015) 370; M. Bauer and M. Neubert, Phys. Rev. Lett. 116 (2016) 141802; R. Barbieri et al, Eur. Phys. J. C 76 (2016) 67; D. Becirevic et al., Phys. Rev. D 94 (2016) 115021; G. Hiller, D. Loose and K. Schonwald, JHEP 1612 (2016) 027.

[15] D. A. Faroughy, A. Greljo and J. F. Kamenik, Phys. Lett. B 764 (2017) 126.

[16] D. Buttazzo, A. Greljo, G. Isidori and D. Marzocca, arXiv:1706.07808 [hep-ph].

[17] S. Fajfer and N. Kosnik, Phys. Lett. B 755 (2016) 270.

[18] Y. Sakaki and H. Tanaka, Phys. Rev. D 87 (2013) 5, 054002; Phys. Rev. D 91 (2015) 114028; M. Duraisamy and A. Datta, JHEP 1309 (2013) 059; D. Becirevic et al., arXiv:1602.03030;

R. Alonso et al, Phys. Rev. D 94 (2016) 094021; M. A. Ivanov et al., Phys. Rev. D 95 (2017) 036021.

[19] P. Colangelo et al., Phys. Rev. D 86 (2012) 054024.

[20] P. Colangelo et al., Phys. Lett. B 293 (1992) 207; Phys. Rev. D 58 (1998) 116005.

[21] C. Patrignani et al. [Particle Data Group], Chin. Phys. C 40 (2016) 100001.

[22] S. Faller, T. Mannel and S. Turczyk, Phys. Rev. D 84 (2011) 014022.

[23] A. Crivellin and S. Pokorski, Phys. Rev. Lett. 114 (2015) 011802.

[24] M. Bordone, G. Isidori and D. van Dyk, Eur. Phys. J. C 76 (2016) 360.

[25] P. Gambino, Int. J. Mod. Phys. A 30 (2015) 1543002.

[26] P. Colangelo and F. De Fazio, Phys. Rev. D 95 (2017) 011701.

[27] J. Chay et al, Phys. Lett. B 247 (1990) 399; I. I. Y. Bigi et al, Phys. Lett. B 293 (1992) 430 Erratum: [Phys. Lett. B 297 (1992) 477]; B. Blok et al, Phys. Rev. D 49 (1994) 3356 Erratum: [Phys. Rev. D 50 (1994) 3572]; A. V. Manohar and M. B. Wise, Phys. Rev. D 49 (1994) 1310.

[28] A. F. Falk, Z. Ligeti, M. Neubert and Y. Nir, Phys. Lett. B 326 (1994) 145.

[29] N. Isgur and M. B. Wise, Phys. Lett. B 237 (1990) 527.

[30] M. Neubert, Phys. Rept. 245 (1994) 259.

[31] I. Caprini, L. Lellouch and M. Neubert, Nucl. Phys. B 530 (1998) 153.

[32] J. A. Bailey et al. [MILC Collaboration], Phys. Rev. D 92 (2015) 034506

[33] J. Bailey et al. [Fermilab Lattice and MILC Collaborations], Phys. Rev. D 89 (2014) 114504.

[34] B. Aubert et al. [BaBar Collaboration], Phys. Rev. D 79 (2009) 012002.

[35] D. Bigi et al., Phys. Lett. B769 (2017) 441; B. Grinstein and A. Kobach, Phys. Lett. B771 (2017) 359.

[36] A. Abdesselam et al. [Belle Collaboration], arXiv:1702.01521 [hep-ex].

[37] C. G. Boyd, B. Grinstein and R. F. Lebed, Phys. Rev. D 56 (1997) 6895.

[38] D. Bigi and P. Gambino, Phys. Rev. D 94 (2016) 094008.

[39] F. U. Bernlochner et al., Phys. Rev. D 95 (2017) 115008. 